\title{
Tissue Distribution and Whole Body Burden of the Chlorinated Polyfluoroalkyl Ether Sulfonic Acid F-53B in Crucian Carp (Carassius carassius): Evidence for a Highly Bioaccumulative Contaminant of Emerging Concern
}

\author{
Yali Shi, ${ }^{\dagger}$ Robin Vestergren, ${ }^{\ddagger}$ Zhen Zhou, ${ }^{\S}$ Xiaowei Song, ${ }^{\dagger}$ Lin $\mathrm{Xu},{ }^{\dagger}$ Yong Liang, ${ }^{\S}, \|$ and Yaqi Cai ${ }^{*}, \dagger, \|$ \\ ${ }^{\dagger}$ State Key Laboratory of Environmental Chemistry and Ecotoxicology, Research Center for Eco-Environmental Science, Chinese \\ Academy of Sciences, Beijing 100085, China \\ ${ }^{\ddagger}$ Department of Environmental Science and Analytical Chemistry (ACES), Stockholm University, Stockholm SE 106 91, Sweden \\ ${ }^{\S}$ Key Laboratory of Optoelectronic Chemical Materials and Devices of Ministry of Education, Jianghan University, Wuhan 430056, \\ China \\ "Institute of Environment and Health, Jianghan University, Wuhan 430056, China
}

\section{Supporting Information}

\begin{abstract}
Following the global actions to phase out perfluoroctanesulfonic acid (PFOS) a large number of alternative per- and polyfluoroalkyl substances, with poorly defined hazard properties, are being used in increasing quantities. Here, we report on the first detection of the chlorinated polyfluoroalkyl ether sulfonic acid F-53B in biological samples and determine the tissue distribution and whole body bioaccumulation factors $\left(\mathrm{BAF}_{\text {whole body }}\right)$ in crucian carp (Carassius carassius). Analysis of fish samples from Xiaoqing River (XR) and Tangxun Lake (TL) demonstrated a similar level of F-53B contamination with median
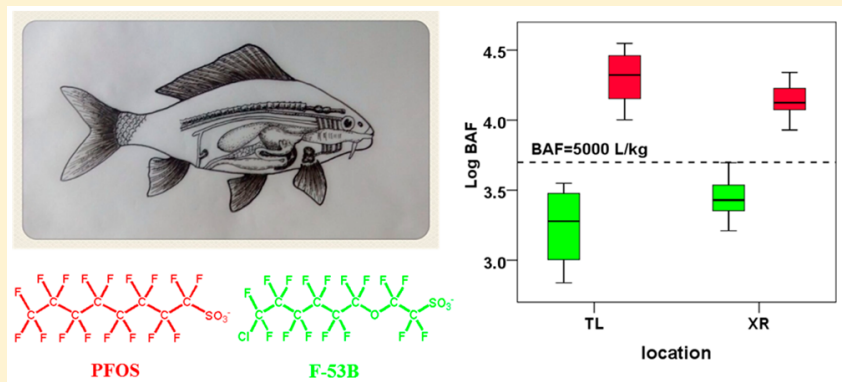

PFOS

F-53B concentrations in blood of 41.9 and $20.9 \mathrm{ng} / \mathrm{g}$, respectively. Tissue/blood ratios showed that distribution of F-53B primarily occurs to the kidney (TL: 0.48, XR: 0.54), gonad (TL: 0.36, XR: 0.54), liver (TL: 0.38, XR: 0.53), and heart (TL: 0.47, XR: 0.47). Median Log $\mathrm{BAF}_{\text {whole body }}$ values for F-53B (XR: 4.124, TL: 4.322) exceeded regulatory bioaccumulation criterion and were significantly higher than those of PFOS in the same data sets (XR: 3.430, TL: 3.279). On the basis of its apparent omnipresence and strong bioaccumulation propensity, it is hypothesized that F-53B could explain a significant fraction of previously unidentified organofluorine in biological samples from China, and regulatory actions for this compound are encouraged.
\end{abstract}

\section{INTRODUCTION}

Per- and polyfluoroalkyl substances (PFASs) comprise a large group of chemicals which have been produced in steadily increasing quantities since the 1950 s. ${ }^{1,2}$ Because of their unique surface tension lowering potential and resistance to degradation, PFASs fill important functions in modern society and are used as water-, oil-, and grease-repellents for packaging, rugs and carpets, and leather; as surfactant detergents, emulsifiers, wetting agents, and dispersants, among other applications. ${ }^{3,4}$ However, since the first reports of perfluorooctane sufonic acid (PFOS) in wildlife ${ }^{5}$ and humans, ${ }^{6}$ concerns regarding the persistence, bioaccumulation potential, and toxic effects of PFASs have triggered regulatory actions and voluntary phaseout initiatives by the fluorochemical industry for this class of chemicals. ${ }^{7}$ In 2000, the major global producer of PFOS announced that it would cease production of perfluorooctane sulfonyl fluoride (POSF) and its derivatives, ${ }^{8}$ and nine years later PFOS was added to Annex B of the Stockholm Convention for Persistent Organic Pollutants (POPs). ${ }^{9}$ In
2006, eight leading global companies also agreed to phase out perfluorooctanoic acid (PFOA) and related chemicals by 2015. ${ }^{10}$ Although the efforts by industry appear to have had a positive effect in some areas of the world, where time trends of PFOS in humans are decreasing, ${ }^{11,12}$ numerous nonregulated replacement chemicals are being produced in increasing quantities. $^{2,13}$

A general strategy of the fluorochemical industry has been to substitute PFOS, PFOA, and their related precursors with short-chain homologues containing 4 or 6 carbon atoms. ${ }^{3}$ Among the large number of alternative PFASs described in the patent literature, there are also many chemical structures with ether linkage(s) between perfluorinated carbons and/or a terminal fluorine atom replaced by chlorine. ${ }^{2,13,14}$ While

Received: September 4, 2015

Revised: November 3, 2015

Accepted: November 11, 2015

Published: November 11, 2015 
perfluoroalkyl ether carboxylic acids (PFECAs) have recently been taken into production to replace PFOA as emulsifiers in polymerization processes, some perfluoroalkyl ether sulfonic acids (PFESAs) have a significantly longer production history. ${ }^{2,14}$ One important example of such an overlooked PFAS is the chlorinated polyfluoroalkyl ether sulfonic acid, F53B (molecular formula $\mathrm{ClCF}_{2} \mathrm{C}_{5} \mathrm{~F}_{10} \mathrm{OCF}_{2} \mathrm{CF}_{2} \mathrm{SO}_{3}{ }^{-}$), which has been applied as a mist suppressant in hard chrome plating for more than 30 years in China. ${ }^{2,14}$ In two recent publications, F-53B was ubiquitously detected in water and sludge samples from different parts of China. ${ }^{14,15}$ Given the structural similarities with PFOS, it has also been hypothesized that F$53 \mathrm{~B}$ may be bioaccumulative and toxic. ${ }^{13,14}$ However, there is currently no data on the uptake and accumulation of this compound in biota.

Previous research has demonstrated that traditional equilibrium approaches based on octanol-water partitioning coefficients $\left(K_{\mathrm{ow}}\right)$ are insufficient to understand the distribution and bioaccumulation of perfluoroalkyl acids. ${ }^{16}$ Unlike most persistent organic pollutants, perfluoroalkyl acids do not partition into storage lipids due to the dipolar nature of the carbon-fluorine bond and their anionic headgroup. ${ }^{16}$ Instead, specific protein-binding mechanisms and/or partitioning to phospholipids have been proposed to explain the surprisingly high bioaccumulation potential of some PFASs. ${ }^{17,18}$ Although significant advances have been made in the development of in silico tools for predicting distribution of accumulation of ionizing and polar chemicals, ${ }^{19,20}$ the application of bioaccumulation models to PFASs relies heavily on empirical data for their parametrization and evaluation. ${ }^{21}$

In the current study, we present a validated analytical methodology for quantification of F-53B in biological samples using high-performance liquid chromatography (HPLC) coupled to Orbitrap high-resolution mass spectrometry (HRMS) and tandem mass spectrometry (MS/MS). The analytical technique was subsequently employed to measure F$53 \mathrm{~B}$ in blood, bile, and eight additional tissues of adult crucian carp and determine the bioaccumulation potential of F-53B under natural environmental conditions. Absolute concentrations, tissue distribution, and bioaccumulation factors (BAFs) of F-53B in crucian carp are presented relative to measurements of linear-PFOS (hereafter referred to simply as PFOS) in the same sample sets.

\section{MATERIALS AND METHODS}

Collection of Fish and Surface Water Samples. Crucian carp (Carassius carassius) was selected as an ideal model species for this study since it is a stationary fresh water species that can live in highly polluted and eutrophic waters and is widely spread throughout China. ${ }^{22}$ Crucian carp were caught from Xiaoqing River $(n=30)$, Shandong province, and Tangxun Lake $(n=13)$, Hubei province, during July 2014. All fish were alive during collection and $2-5 \mathrm{~mL}$ whole blood samples $(n=$ 43) were drawn using syringe, rinsed with a solution of heparin sodium, and then transferred to a BD Vacutainer tube (Becton Dickinson Vacutainer System, UK) with EDTA. The fish were carefully dissected to get liver $(n=43)$, kidney $(n=43)$, brain $(n=43)$, heart $(n=42)$, swim bladder $(n=43)$, gill $(n=43)$, gonad $(n=43)$, muscle $(n=43)$, and bile $(n=29)$ samples after being transported to the laboratory. All tissue samples, except bile, were weighed after being cleaned with purified water in order to remove excess blood, and were subsequently homogenized and kept at $-20{ }^{\circ} \mathrm{C}$ until further extraction. The length, weight, and sex of the fish was recorded along with the weight of all individual tissues except blood, muscle, and bile (see Supporting Information, Table S1). Surface water samples $(n=5)$ were collected at approximately $1 \mathrm{~m}$ depth in parallel with the fish samples using precleaned polypropylene beakers. In addition to the fish samples from Tangxun Lake and Xiaoqing River, crucian carp $(n=10)$ were purchased from a market in Beijing for analytical method development purposes. After dissection, following the same protocol as above, the market fish samples were homogenized and pooled.

Reagents and Standards. Native and mass-labeled linear PFOS standard solutions were purchased from Wellington laboratories. An authentic F-53B standard $\left({ }^{19} \mathrm{~F}-\mathrm{NMR}\right.$ result shown in Figure S1) was purified from the commercial F-53B product purchased from Shanghai Synica Co., Ltd. Methanol (HPLC grade) was obtained from Fisher Scientific (Pittsburgh, PA, USA). Ammonium acetate $\left(\mathrm{NH}_{4} \mathrm{OAc},>97 \%\right)$, ammonium hydroxide (v/v, 50\%, HPLC grade), tetra- $n$-butylammonium hydrogen sulfate (TBA, 97\%), and methyl tert-butyl ether (MTBE, >99\%) were obtained from Alfa Aesar (Ward Hill, MA, USA). Potassium hydroxide, sodium carbonate, and sodium bicarbonate (reagent grade, RG) were purchased from Beijing Chemical Works. Glass-fiber filter membranes $(0.45 \mu \mathrm{m}, 47 \mathrm{~mm})$ and Oasis WAX solid-phase extraction cartridges $\left(150 \mathrm{mg}, 6 \mathrm{~cm}^{3}\right)$ were purchased from Sartorius Stedim Biotech (Goettingen, Germany) and Waters Co. (Milford, MA, USA), respectively. All ultrapure water ( $>18.2$ $\mathrm{M} \Omega \mathrm{cm}^{-1}$ ) used in the present study was prepared with a MilliQ Advantage A10 system (Millipore, Billerica, MA, USA).

Sample Treatment. The methods for extraction and cleanup were similar to methods previously developed for PFOS and other anionic PFASs. ${ }^{5,6}$ In brief, $200 \mathrm{~mL}$ of water, prefiltered through a $0.45-\mu \mathrm{m}$ glass fiber filter membrane, was spiked with $2 \mathrm{ng}$ of internal standard $\left({ }^{13} \mathrm{C}_{4}-\mathrm{PFOS}\right)$ and then loaded onto an Oasis WAX single-use cartridge $(6 \mathrm{cc} / 150 \mathrm{mg})$, which was preconditioned using $4 \mathrm{~mL}$ of $0.1 \%$ ammonium hydroxide (in methanol), $4 \mathrm{~mL}$ of methanol, and $4 \mathrm{~mL}$ of ultrapure water at a rate of $1 \mathrm{drop} / \mathrm{s}$. The cartridges were subsequently washed using $4 \mathrm{~mL}$ of buffer solution $(25 \mathrm{mmol} /$ $\mathrm{L}$ acetic acid/ammonium acetate, $\mathrm{pH}=4)$ and $8 \mathrm{~mL}$ of ultrapure water and centrifuged for $10 \mathrm{~min}$ at $3000 \mathrm{rpm}$ to remove the residual water. Finally, the target compounds were eluted using $4 \mathrm{~mL}$ of methanol and $4 \mathrm{~mL}$ of $0.1 \%$ ammonium hydroxide (in methanol), which was concentrated to $1 \mathrm{~mL}$ under nitrogen gas prior to injection.

Homogenized fish muscle samples were freeze-dried under vacuum and then extracted using an alkaline digestion method. ${ }^{23}$ Briefly, $0.2 \mathrm{~g}$ of muscle sample (dry weight) was spiked with $2 \mathrm{ng}$ of internal standard and sonicated in $10 \mathrm{~mL}$ of $10 \mathrm{mmol} / \mathrm{L} \mathrm{KOH}$ methanol solution at $60{ }^{\circ} \mathrm{C}$ for $30 \mathrm{~min}$, followed by shaking at $250 \mathrm{rpm}$ for $16 \mathrm{~h}$. The supernatant was transferred and concentrated to $1 \mathrm{~mL}$ under nitrogen gas. The concentrated extract was diluted to about $50 \mathrm{~mL}$ using ultrapure water and loaded onto a WAX cartridge for cleanup following the same procedure as for water samples.

Homogenized fish blood, bile, and tissue samples were extracted using an ion-pair liquid-liquid extraction method, ${ }^{6}$ followed by SPE cleanup. ${ }^{23,24}$ Briefly, $0.2 \mathrm{~g}$ of sample material was spiked with $2 \mathrm{ng}$ of internal standard, $2 \mathrm{~mL}$ of $\mathrm{NaHCO}_{3} /$ $\mathrm{Na}_{2} \mathrm{CO}_{3}$ buffer solution ( $\left.\mathrm{pH}=10\right)$, and $1 \mathrm{~mL}$ of TBA solution, and then extracted using $5 \mathrm{~mL}$ of MTBE by shaking at $250 \mathrm{rpm}$ for $20 \mathrm{~min}$. Approximately $4.5 \mathrm{~mL}$ of supernatant was transferred to a $15-\mathrm{mL}$ polypropylene centrifugal tube and 
the residue was extracted two more times with $5 \mathrm{~mL}$ of MTBE. The combined extracts were subsequently concentrated to $\sim 0.5$ $\mathrm{mL}$ under nitrogen gas. The concentrated extract was diluted to $1 \mathrm{~mL}$ using methanol and then to $50 \mathrm{~mL}$ using ultrapure water, which was loaded onto a WAX cartridge for cleanup following the same procedure as for water samples.

Instrumental Analysis. HPLC-Orbitrap MS was used to identify F-53B in blood, bile, and tissue samples, whereas quantification was performed by HPLC-MS/MS. Orbitrap Fusion MS system (Thermo Fisher Scientific Inc., Waltham, $\mathrm{MA}$ ), was operated in negative electrospray ionization (ESI). Data were acquired in the full scan mode $(200-700 \mathrm{~m} / z)$ with a resolution of 120000 . The detailed parameters for Orbitrap MS were as follows: capillary voltage, $2500 \mathrm{~V}$; ion transfer tube and vaporizer temperature, 350 and $200{ }^{\circ} \mathrm{C}$; the higher-energy collision dissociation (HCD) energy, 35\%. Chromatographic separation was achieved using an Acclaim $120 \mathrm{C} 18$ column (5 $\mu \mathrm{m}, 4.6 \mathrm{~mm} \times 150 \mathrm{~mm})$ and a mobile phase of methanol (eluent A) and $50 \mathrm{mM}$ ammonium acetate in water (eluent B). The solvent gradient started at $28 \% \mathrm{~B}$, ramped over 4 min to $5 \% \mathrm{~B}$, held for $3 \mathrm{~min}$ at $5 \% \mathrm{~B}$, increased to $28 \% \mathrm{~B}$, and held at this level until $10 \mathrm{~min}$. The flow rate was at $1.0 \mathrm{~mL} / \mathrm{min}$.

Quantitative analysis of F-53B and PFOS in all samples was achieved by HPLC (Ultimate 3000, Thermo Fisher Scientific Co.) electrospray ionization MS/MS (API 4500, Applied Biosystems/MDS SCIEX, USA). The HPLC instrument was equipped with a dual pump (HPG-3200RS), autosampler (WPS-3000RS), column compartment (TCC-3000RS), and DCMS Link software. The experimental conditions for ESIMS/MS were optimized as follows: curtain gas pressure, $25 \mathrm{psi}$; collision gas pressure, $3 \mathrm{psi}$; ion spray voltage, $-2000 \mathrm{~V}$; temperature, $400{ }^{\circ} \mathrm{C}$; gas 1:50 psi and gas 2:40 psi. The detailed MS transition and parameters for F-53B and PFOS are shown in Table S2. A $10-\mu \mathrm{L}$ sample aliquot was injected on an Ascentis Express F5 PFP Column $(2.7 \mu \mathrm{m}, 90 \AA, 10 \mathrm{~cm} \times 2.1$ $\mathrm{mm}$, Sigma-Aldrich) for separation and analysis of PFOS and F53B. The mobile phase consisted of methanol (eluent A) and $20 \mathrm{mM}$ ammonium hydroxide $/ 20 \mathrm{mM}$ formic acid in water $(\mathrm{pH}=4$, eluent $\mathrm{B})$. The solvent gradient started at $90 \% \mathrm{~B}$, then ramped to $40 \% \mathrm{~B}$ by $1.0 \mathrm{~min}$ and held for $2 \mathrm{~min}$, decreased to $12 \% \mathrm{~B}$ by $14 \mathrm{~min}, 0 \% \mathrm{~B}$ by $14.5 \mathrm{~min}$, returned to initial conditions by $14.6 \mathrm{~min}$, and held for $6.4 \mathrm{~min}$. The flow rate was constant at $0.25 \mathrm{~mL} / \mathrm{min}$.

Quantification was performed by the internal standard approach using ${ }^{13} \mathrm{C}_{4}$-PFOS for both PFOS and F-53B. A 9point calibration curve $(0.05,0.1,0.5,1.0,5,10,20,50$, and 100 $\mu \mathrm{g} / \mathrm{L})$ was established using $1 / x^{2}$ weighted regression $\left(r^{2}>\right.$ 0.99). The matrix-specific limits of quantification (LOQ) were defined as the lowest concentration resulting in a signal-tonoise ratio $(\mathrm{S} / \mathrm{N}) \geq 10$ (details are shown in Table S3).

Quality Assurance. All laboratory consumables, solvents, and dissection tools were checked for contamination, and one procedural blank sample was conducted for every ten samples. Field blanks included polypropylene bottles filled with milli-Q water. However, all procedural, field, and solvent injection blanks were consistently below instrumental detection limits. Instrumental drift was monitored by injecting a calibration standard for every 10 sample injections and a new calibration curve was constructed if a deviation of more than $\pm 20 \%$ from its initial value was observed. Spike-recovery experiments $(n=$ 4) were used to determine accuracy and precision for all individual fish tissues and surface water. Briefly, $2 \mathrm{ng}$ of PFOS and F-53B were added to blood, bile, and tissue homogenates from the market fish samples collected in Beijing. For water samples, tap water was used as a test matrix for method development. After fortification, the test matrices were treated according to the same procedure as real samples.

Bioaccumulation Metrics. Tissue/blood ratios, whole body burdens, and BAFs were calculated to describe the distribution and accumulation of F-53B and PFOS in crucian carp. Tissue/blood ratios were defined as the concentration of chemical in a given tissue over the concentration in blood on a fresh weight basis. The whole body burden (ng) was calculated according to eq 1

$$
\text { whole body burden }=m_{\text {fish }} \sum_{n=1}^{i} C_{\text {tissue }, n} \times f_{\text {tissue }, n}
$$

where $m_{\mathrm{fish}}$ is the total body weight $(\mathrm{g}), C_{\text {tissue }}$ is the concentration of chemical in tissue $(\mathrm{ng} / \mathrm{g})$, and $f_{\text {tissue }}$ is the mass fraction of individual tissues (unitless). Results of the whole body burden were subsequently used to calculate the percentage of chemical present in different tissues. BAFs were calculated using (i) single tissue concentrations $\left(\mathrm{BAF}_{\text {tissue }}\right)$ (eq 2) and (ii) whole body concentration ( $\left.\mathrm{BAF}_{\text {whole body }}\right)$, respectively.

$$
\mathrm{BAF}_{\text {tissue }}=\frac{C_{\text {tissue }}}{C_{\text {water }}}
$$

For calculation of whole body BAFs, $C_{\text {tissue }}$ was substituted with $C_{\text {whole body, }}$ which was defined according to eq 3 .

$$
C_{\text {whole body }}=\sum_{n=1}^{i} C_{\text {tissue }, n} \times f_{\text {tissue }, n}
$$

Tissue/blood ratios, whole body burdens, and BAFs were calculated for every individual fish on the basis of specific tissue concentrations and body fractions from post-mortem examinations (Table S4). Because blood and muscle weights were not recorded during dissection, their body fractions were estimated as 0.045 and 0.5 , respectively, based on literature values in other fish species. ${ }^{25,26}$ Bile was excluded from the whole body burden and BAF calculations due to its negligible body fraction. The concentration of chemical in the remaining carcass, which was not measured here, was assumed to be half of that in muscle tissue. Although there may be additional tissues that can accumulate PFASs, a conservatively low fraction of chemical in the rest of body compared to previous studies ${ }^{27,28}$ was assumed for the carcass in order not to overestimate the $\mathrm{BAF}_{\text {whole body. }}$

Statistical Analysis. Statistical analysis was performed using IBM PASW statistics 18.0 (SPSS Inc., 1993-2007) with a statistical significance threshold of $p<0.05$. The nonparametric Mann-Whitney U-test was used to test for differences in tissue/blood ratios between F-53B and PFOS and to test for differences in whole body BAFs between F-53B and PFOS for the two different sampling locations. Spearman's rank correlation analysis was used to examine relationships between whole body BAFs, body weight, length, and body fraction of tissues.

\section{RESULTS AND DISCUSSION}

Identification and Quantification of F-53B. Although MS/MS is typically the method of choice for analysis of known PFASs in environmental samples, coeluting endogenous molecules have previously been observed to produce false positives for perfluoroalkanesulfonic acids in biological 
a: F-53B standard $(50 \mu \mathrm{g} / \mathrm{L})$
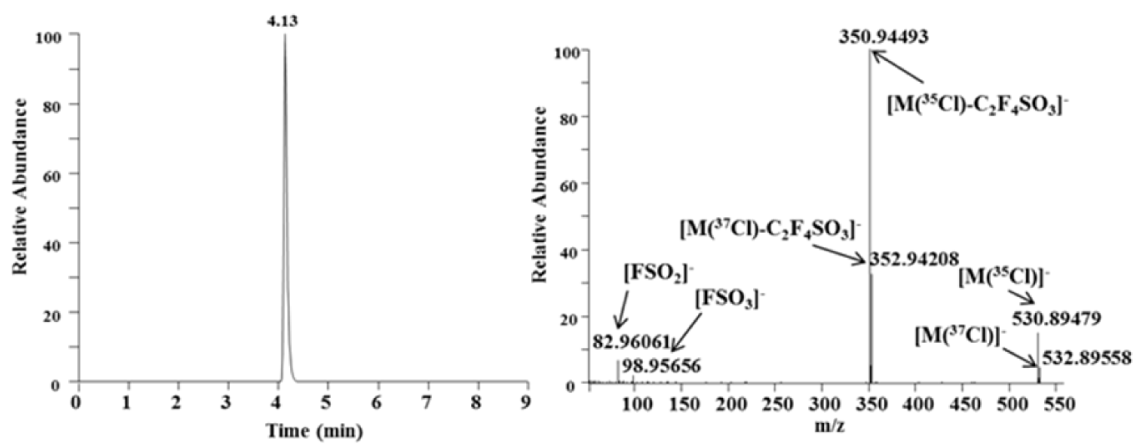

b: Tissue sample
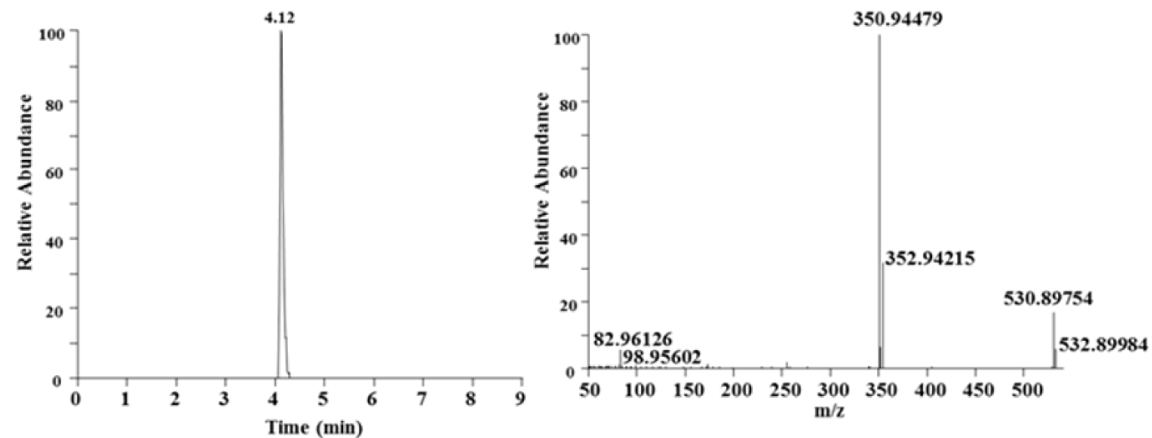

c: Reconstructed total ion chromatography

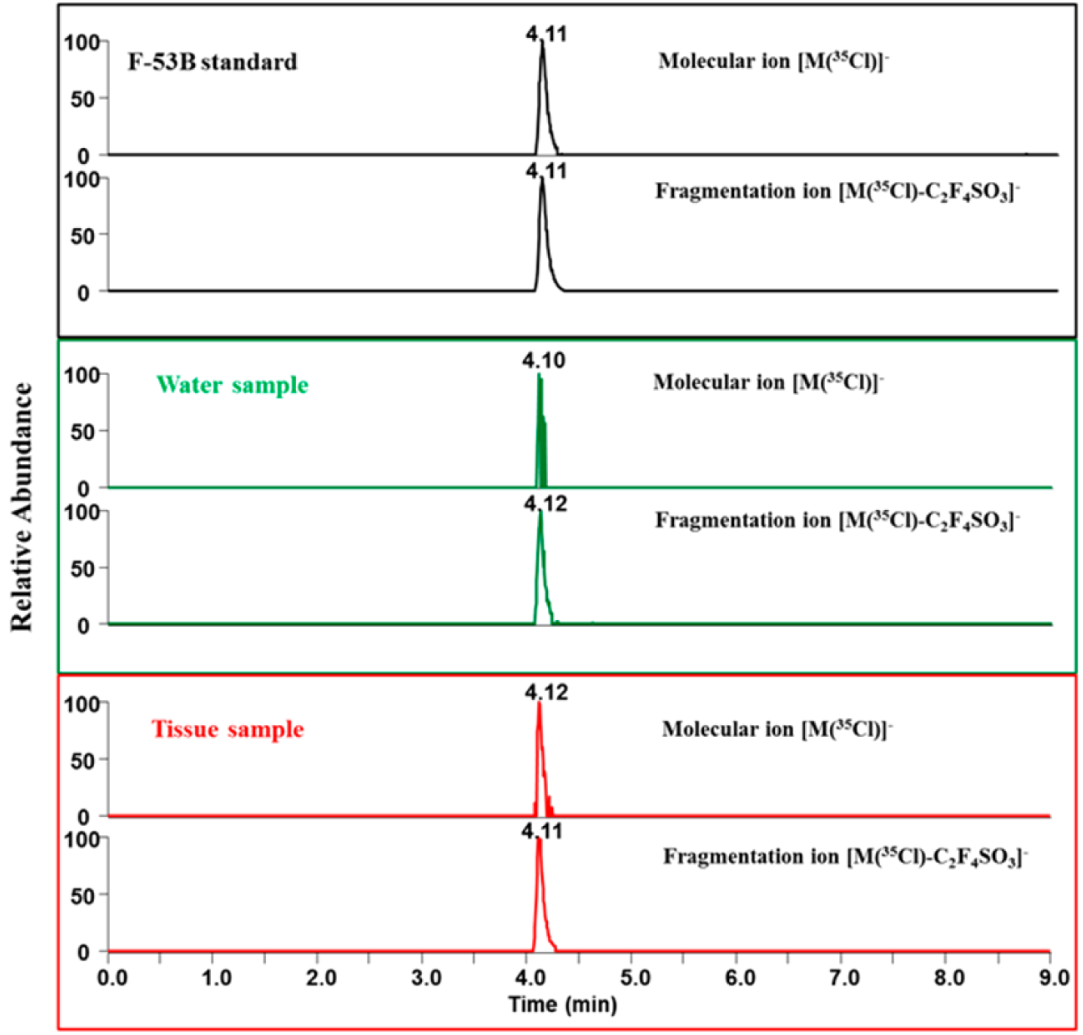

Figure 1. Chromatogram and mass-spectra of F-53B in a standard solution, water, and fish tissue extract using Orbitrap high HRMS. (a) F-53B standard $(50 \mu \mathrm{g} / \mathrm{L}), m / z: 530.89558$ (left: full scan, right: $\mathrm{MS}^{2}$ ions); (b) a tissue sample, $m / z: 530.89558$ (left: full scan, right: $\mathrm{MS}^{2}$ ions); (c) reconstructed total ion chromatography of the molecular and fragmentation ion transits of F-53B in standard solution $(50 \mu \mathrm{g} / \mathrm{L})$, water, and tissue sample with mass windows of theoretical mass of $\pm 5 \mathrm{ppm}$.

samples. ${ }^{29}$ In the present study we utilized the high mass accuracy and spectral resolving power of Orbitrap HRMS to investigate interferences and confidently identify F-53B. Figure
1 depicts HRMS spectra and chromatogram of F-53B in a standard solution, water, and fish tissue extracts. The major fragmentation ions, $\left[\mathrm{M}\left({ }^{35} \mathrm{Cl}\right)\right]^{-},\left[\mathrm{M}\left({ }^{35} \mathrm{Cl}\right)-\mathrm{C}_{2} \mathrm{~F}_{4} \mathrm{SO}_{3}\right]^{-}$, 

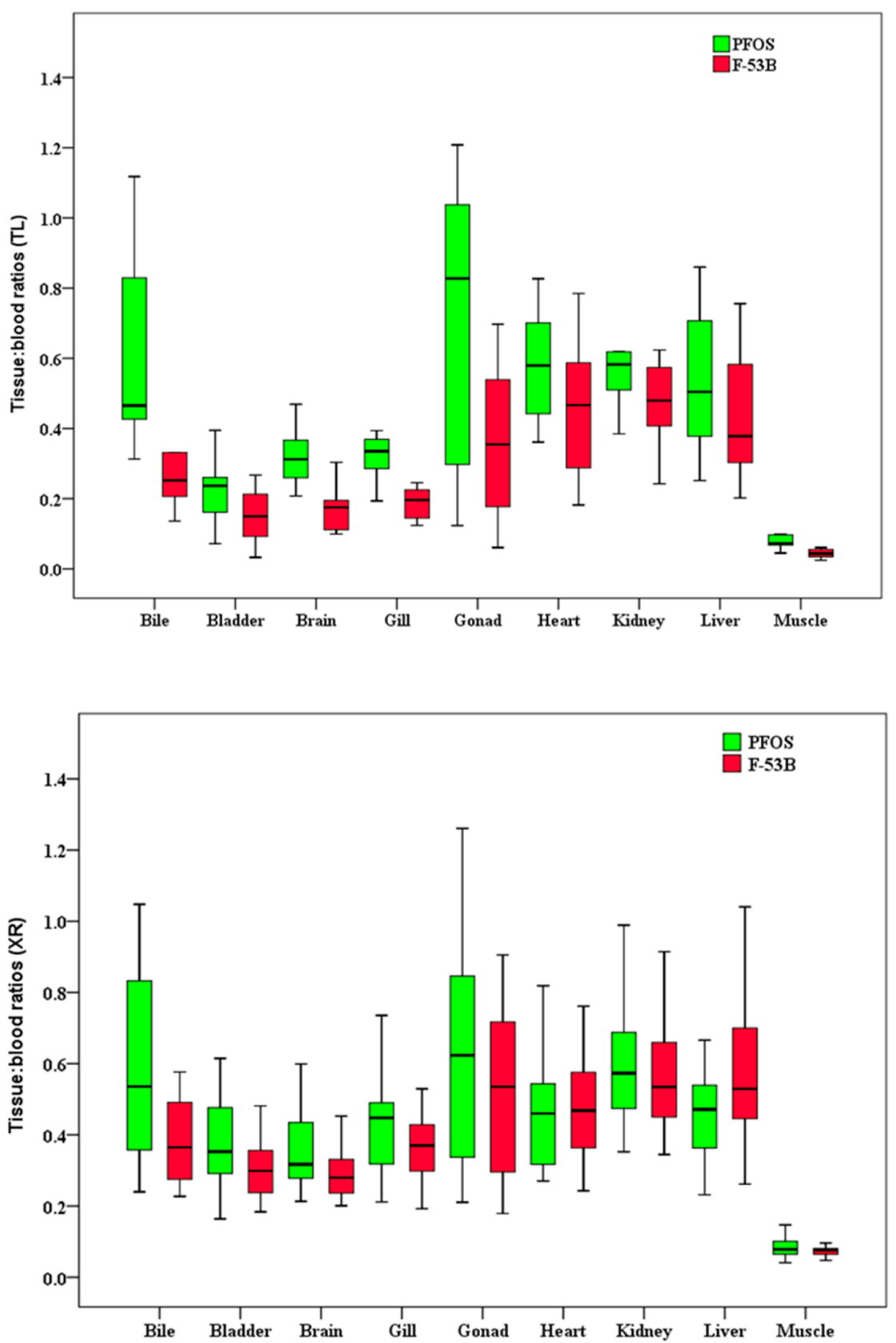

Figure 2. Box and whisker plots of tissue/blood ratios for PFOS and F-53B in Tangxun Lake (TL) and Xiaoqing River (XR), respectively. The horizontal line in the box represents the median, and the lower and upper edges of the box mark the 25th and 75th percentiles, respectively. The whiskers extending from the box represent the maximum and minimum values excluding outliers. A value of $p<0.05$ was considered to indicate a significant difference in different ratios.

$\left[\mathrm{FSO}_{2}\right]^{-}$, and $\left[\mathrm{FSO}_{3}\right]^{-}$, observed in HRMS MS ${ }^{2}$ full scan mode for tissue sample, were identical to those in the F-53B standard solution, with similar relative abundance and isotopic ratios of ${ }^{35} \mathrm{Cl}$ to ${ }^{37} \mathrm{Cl}$ (about 3:1) (shown in Figure 1a and b). The observation of the molecular ion $(\mathrm{m} / z=530.89754 ; \Delta m=$ $-0.593 \mathrm{ppm})$ and characterized ion $(\mathrm{m} / z=350.94479 ; \Delta m=$ $1.917 \mathrm{ppm})$ in tissue samples at identical retention times with those of standard solutions further confirmed the presence of F53B (Figure 1c). Interestingly, the characteristic ions of the tencarbon $\mathrm{Cl}$ PFESA homologue (molecular formula $\mathrm{ClCF}_{2} \mathrm{C}_{7} \mathrm{~F}_{12} \mathrm{OCF}_{2} \mathrm{CF}_{2} \mathrm{SO}_{3}{ }^{-} ; m / z=630.88751 ; \Delta m=-0.931$ ppm and $m / z=450.93820 ; \Delta m=1.175 \mathrm{ppm})^{15}$ were also observed at a retention time of $4.91 \mathrm{~min}$ (Figure S2). However, due to low absolute intensity and lack of authentic standards we did not further investigate the tentative detection of other $\mathrm{Cl}$ PFESA homologues.

Quantification using MS/MS demonstrated good agreement in absolute intensity ratios of the quantifier ion $(530.6>351.0)$ and qualifier ion $(530.6>83.0)$ with tissue and water samples deviating less than $8 \%$ from that of the standard solution (Table S5). Fortified water and tissue samples displayed acceptable to excellent recoveries $(92.9 \pm 6.2 \%$ for F-53B and $99.1 \pm 6.9 \%$ for PFOS in water, from $71.0 \pm 9.9 \%$ to $109.2 \pm 5.1 \%$ for F$53 \mathrm{~B}$ and from $75.6 \pm 2.6 \%$ to $103.9 \pm 4.2 \%$ for PFOS in different tissue and body fluid) (shown in Table S3). When optimizing the chromatographic conditions for F-53B, both $\mathrm{C} 18$ and PFP stationary phases were tested for their performance. Although both types of columns provided 
PFOS (TL)


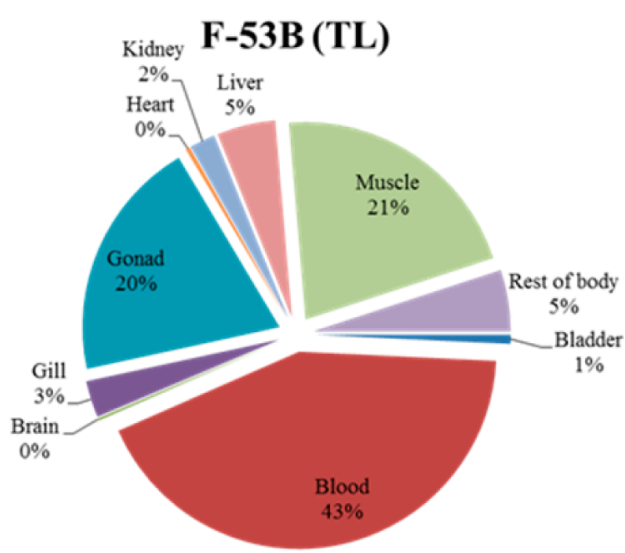

F-53B (XR)

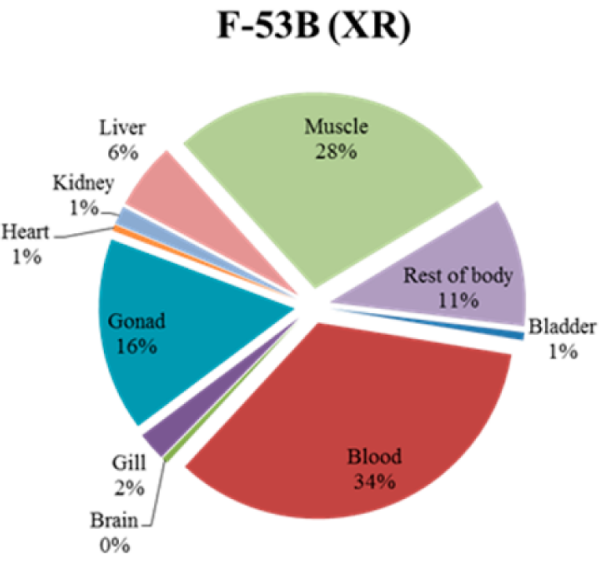

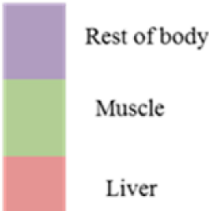

Kidney

Heart

Gonad

Gill

Brain

Blood

Bladder

Figure 3. Whole body burden of PFOS and F-53B in crucian carp from Tangxun Lake (TL) and Xiaoqing River (XR) expressed as percentage of the total amount of chemical present in different tissues.

excellent peak shapes for F-53B (see Figures S3-S5), the fluorinated stationary phase was chosen due to its ability to effectively separate PFAS isomers and reduce matrix suppression/enhancement effects. ${ }^{29,30}$ When comparing the chromatograms of F-53B and PFOS, it is noteworthy that only one peak, belonging to the linear isomer, was observed for F$53 \mathrm{~B}$ in water and tissue samples from both sampling locations (Figures S6 and S7). The absence of branched isomers confirms the assumption by Wang et al. ${ }^{31}$ that F-53B, unlike PFOS, is derived from telomer-based raw materials resulting in an isomerically pure product. ${ }^{30}$ Overall, the analytical method validation demonstrates that F-53B can be confidently quantified in complex biological matrices using methodologies similar to those for other anionic PFASs. However, native and mass-labeled standards for Cl PFESAs are needed to improve analytical accuracy and expand the quantitative analysis to a wider range of compounds.

Concentrations in Surface Water and Fish Tissues. Both F-53B and PFOS were detected above the LOQ in all tissue and surface water samples (see Table S6 and Figure S8). In surface water, F-53B was found at quite low and similar concentrations in Xiaoqing River (arithmetic mean (AM) 0.419 ng/L) and Tangxun Lake (AM 0.149 ng/L). Contrastingly, PFOS concentrations varied more than 2 orders of magnitude between Xiaoqing River (AM $2.097 \mathrm{ng} / \mathrm{L}$ ) and Tangxun Lake (AM $242.7 \mathrm{ng} / \mathrm{L}$ ). The substantially elevated concentrations of PFOS in Tangxun Lake have previously been attributed to the direct release from POSF production sources, ${ }^{23,24}$ whereas the contamination profile of PFOS along Xiaoqing River was likely influenced by multiple diffuse sources. ${ }^{32}$ Although concomitantly high concentrations of F-53B and PFOS have been observed in wastewater from metal plating facilities in China, ${ }^{14}$ the differences in F-53B to PFOS ratios between Xiaoqing River and Tangxun Lake indicate that differences in their production sources may also lead to variability in their emission patterns and geographical distribution. A comparison with surface water concentrations of F-53B downstream of municipal wastewater facilities $(10-50 \mathrm{ng} / \mathrm{L})^{14}$ further suggests that the concentrations in both Xiaoqing River and Tangxun Lake reflect relatively low levels of contamination.

Concentrations of F-53B in fish samples varied greatly among the different tissues, with the greatest concentrations in blood (Figure S8). Median blood concentrations of F-53B were 20.9 and $41.9 \mathrm{ng} / \mathrm{g}$ in Tangxun Lake and Xiaoqing River, respectively. Interestingly, F-53B was also quantified in the fish tissue homogenates $(27.8 \mathrm{ng} / \mathrm{g}$ in blood) used for method development purposes obtained from a Beijing fish market (Table S7). The omnipresence of F-53B at similar concentrations in crucian carp from 3 different areas of China supports previous findings that this contaminant is widely distributed around China $^{14,15}$ and indicates that consumption of fish is a likely exposure pathway for humans. Concentration profiles of PFOS in fish tissues followed a pattern similar to that of F-53B, with the highest levels in blood (Figure S8). However, the concentrations in Tangxun Lake samples were 2 orders of magnitude higher than those from Xiaoqing River, reflecting the higher exposure concentrations in surface water. 
Tissue Distribution and Whole Body Burden. The distribution into different tissues after uptake largely determines the fate of a chemical and is of great importance for understanding the underlying mechanisms of bioaccumulation. ${ }^{20,21}$ In Figure 2 and Table S8, the tissue/blood ratios for F-53B are presented and compared to those of PFOS in fish samples from Tangxun Lake (TL) and Xiaoqing River (XR), respectively. Overall, similar tissue/blood ratios were observed for F-53B and PFOS, suggesting that these compounds share similar mechanisms of distribution. The finding of blood as a primary body compartment for F-53B is broadly consistent with the previous findings of several perfluoroalkyl acids binding to serum albumin. ${ }^{33-36}$ Relatively high median tissue/ blood ratios of F-53B in kidney (TL: 0.48, XR: 0.54), gonad (TL: 0.36, XR: 0.54), liver (TL: 0.38, XR: 0.53), and heart (TL: 0.47, XR: 0.47) further suggest that additional sorption to hepatic fatty acid binding proteins ${ }^{37,38}$ and/or partitioning to phospholipids ${ }^{39-41}$ plays an important role in the distribution of F-53B. Because the protein- and phospholipid composition of specific tissues was not measured here, it is difficult to delineate between these different mechanisms of tissue distribution. Nevertheless, it is noteworthy that gonad and swim bladder tissue, which have a high phospholipid content, ${ }^{42,43}$ also displayed relatively high tissue/blood ratios for both F-53B and PFOS, adding some support for this accumulation mechanism. ${ }^{18}$ The median tissue/blood ratios for F-53B in bile (TL: 0.25, XR: 0.37) further suggest that secretion from the liver ${ }^{44}$ may be an important clearance mechanism for this chemical. However, since it is likely that F53B, analogously to PFOS, undergoes enterohepatic circulation, the blood to bile ratios cannot be used to calculate biliary clearance rates. ${ }^{45}$

Despite that some of the highest tissue/blood ratios were observed for liver, kidney, and heart, the combined contribution of these organs to the whole burden of F-53B was fairly small $(<10 \%)$ when the body fraction of tissues was considered (Figure 3). In fact, $>75 \%$ of the whole body burden of both F$53 \mathrm{~B}$ and PFOS was present in blood, muscle, and gonad. The relatively large fraction of F-53B (TL: 19.9\%, XR: 15.6\%) and PFOS (TL: 22.6\%, XR: 19.6\%) stored in gonads represents an interesting observation since only a small number of studies have previously investigated the distribution of PFASs to this organ. ${ }^{28,46,47}$

Although the tissue distribution patterns of F-53B and PFOS were qualitatively similar and fairly consistent between the sampling sites, some subtle differences in tissue/blood ratios could be observed in the statistical analysis (see Table S9). Statistically significantly higher tissue/blood ratios for PFOS compared to F-53B were found in all tissues, except heart, kidney, and liver in the Tangxun Lake data set. Contrastingly, only the tissue/blood ratios of PFOS in brain and gill were significantly higher than those of F-53B for the Xiaoqing River data (Mann-Whitney U-test, $P<0.05)$. When comparing the tissue distribution for individual compounds between the two data sets, we found higher tissue/blood ratios of F-53B in in bladder, gill, brain, gonad, liver, and muscle from Xiaoqing River compared to Tangxun Lake (Mann-Whitney U-test, $P<$ 0.05 ). For PFOS, bladder and gill displayed statistically significantly higher tissue:blood ratios from Xiaoqing River, whereas a significantly higher tissue/blood ratio was observed in heart from Tangxun Lake (Mann-Whitney U-test, $P<$ 0.05 ). The above observations can probably be attributed to a combination of physiological differences and/or differences in the exposure conditions between the two sampling sites. For instance, it is likely that the protein and phospholipid content in organs such as liver, gonads, and muscle varies greatly with the feeding status and sexual maturity of fish. ${ }^{42,48}$ Thus, the uptake and sorption of perfluoroalkyl acids to different tissues may also display some variation even within the same species. It is also possible that the high water concentrations of PFOS in Tangxun Lake partly saturate the binding sites on serum albumin allowing the chemical to distribute more to other tissues at higher exposure concentrations. ${ }^{49,50}$

Tissue-Specific and Whole Body BAFs. The BAF of a chemical in fish is a central concept in hazard assessment that describes the net result of competing uptake and elimination processes under natural conditions. ${ }^{51}$ Because of the lack of a suitable normalization criteria for PFASs, such as total lipid weight for neutral organic chemicals, BAFs have sometimes been reported for individual tissues. ${ }^{16}$ Median $\log \mathrm{BAF}_{\text {tissue }}$ values for F-53B varied considerably among the different tissues, ranging from 3.80 to 5.23 for TL, and 3.92 to 5.01 for $\mathrm{XR}$, but were consistently higher than ones for PFOS (see also Table S10). However, for assessment of the bioaccumulation potential compared to regulatory criteria, whole body concentrations should be used because concentrations in most measured tissues would overestimate bioaccumulation potential. In Figure 4, Log $\mathrm{BAF}_{\text {whole body }}$ values are presented for

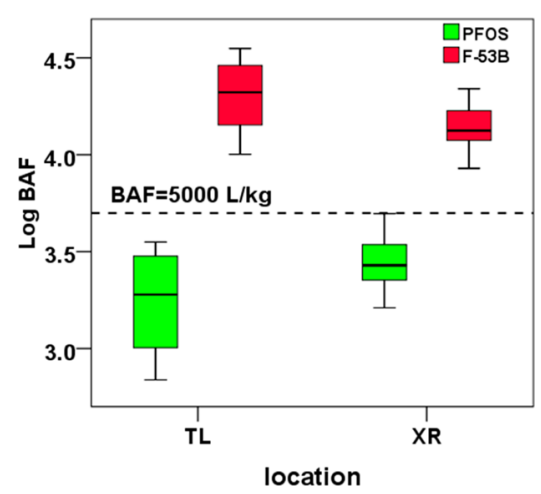

Figure 4. Box and whisker plots of whole body BAFs for PFOS and F$53 \mathrm{~B}$ in crucian carp from Tangxun Lake (TL) and Xiaoqing River (XR). The horizontal line in the box represents the median, and the lower and upper edges of the box mark the 25th and 75th percentiles, respectivelhy. The whiskers extending from the box represent the highest and lowest values, excluding outliers. A value of $p<0.05$ was considered to indicate a statistically significant difference in BAFs.

F-53B and PFOS in comparison to the common regulatory bioaccumulation criterion $\mathrm{BAF}=5000 \mathrm{~L} / \mathrm{kg}^{51}{ }^{51}$ Median Log $\mathrm{BAF}_{\text {whole body }}$ values for F-53B (XR: 4.124, TL: 4.322) greatly exceeded the regulatory bioaccumulation criterion and were statistically significantly higher than those for PFOS (XR: 3.430, TL: 3.279) (Mann-Whitney U-test, $p<0.05$ ). Previous studies on PFASs have observed a positive relationship between the perfluoroalkyl chain-length and BAFs in fish, with the highest bioaccumulation propensity for the hydrophobic longchain perfluoroalkyl carboxylic acids. ${ }^{16,21,27,28,46,47}$ However, a comparison with previous studies demonstrates that F-53B displays $\mathrm{BAF}_{\text {whole body }}$ values similar to or even higher than those of perfluorododecanoic acid. ${ }^{16,28}$ The higher BAFs of F-53B compared to PFOS can partly be explained by its larger molecular volume (407.91-413.61 $\AA$ compared to $381.77 \AA$ ) and hence increasing hydrophobicity. ${ }^{13,14}$ However, while the 
difference in $K_{\mathrm{ow}}$ of the nondissociated form of PFOS and F$53 \mathrm{~B}$ has been estimated to be $0.6 \log$ units, ${ }^{13}$ the difference in median BAFs from Tangxun Lake was more than 1 log unit (see Table S10). The $\mathrm{BAF}_{\text {whole body }}$ determined here was also 0.3-0.5 Log units higher than predicted by Wang et al. using the physical-chemical property estimation software EPI suite. ${ }^{14}$ Collectively, these comparisons indicate that specific protein binding mechanisms related to the uptake and/or elimination may contribute to the high bioaccumulation potential of F-53B. For instance, it is possible that the insertion of an ether linkage increases the flexibility of the fluorinated chain making it fit better into hydrophobic binding pockets. ${ }^{21}$

A comparison of the two sampling sites revealed statistically significantly higher median $\mathrm{BAF}_{\text {whole body }}$ values for $\mathrm{F}-53 \mathrm{~B}$ in Tangxun Lake compared to Xiaoqing River (Mann-Whitney U-test; $p<0.05)$. For PFOS, the opposite trend was observed with statistically significantly higher $\mathrm{BAF}_{\text {whole body }}$ values in Xiaoqing River compared to those in Tangxun Lake (MannWhitney U-test; $p<0.05$ ). The lower $\mathrm{BAF}_{\text {whole body }}$ of PFOS in Tangxun Lake compared to that in Xiaoqing river is an interesting observation, as it may indicate that the bioaccumulation potential is concentration-dependent. ${ }^{50}$ As discussed above, a partial saturation of serum albumin at higher exposure concentrations may lead to a higher fraction of unbound chemical that can be distributed to other tissues. At the same time, a larger fraction of unbound chemical in serum would also lead to an increased elimination rate and, hence, a lower $\mathrm{BAF}_{\text {whole body. }}{ }^{52}$ Yet, other factors related to both exposure conditions and physiological parameters may also contribute to the observed differences between the sampling sites.

To investigate the influence of physiological parameters on the bioaccumulation potential of F-53B and PFOS, Spearman's rank correlations between body weight, length, and body fractions of different organs and $\mathrm{BAF}_{\text {whole body }}$ values were tested (Tables S11 and S12). A weak negative correlation between BAF $_{\text {whole body }}$ and length was observed for PFOS in Xiaoqing river (Spearman's $\rho=-0.496 ; p<0.01$ ), but the correlations were not consistently observed in the Tangxun Lake data set and no correlations with length or weight were observed for F53B. In contrast, moderate to strong correlations were consistently observed between the body fraction of gonads and $\mathrm{BAF}_{\text {whole body }}$ for both F-53B and PFOS in both the Xiaoqing river (Spearman's $\rho>0.404 ; p<0.05$ ) and Tangxun lake data sets (Spearman's $\rho>0.658 ; p<0.05$ ). Considering that gonads were estimated to be the third most important repository of F-53B and PFOS on a whole body basis (Figure 3 ), it seems reasonable that the positive correlation between body fraction of gonad and a higher $\mathrm{BAF}_{\text {whole body }}$ reflects an increasing storage capacity of chemical. However, because the gonadal status is closely linked to sexual maturity and hormonal status of fish, ${ }^{48}$ the correlation between $\mathrm{BAF}_{\text {whole body }}$ and gonadal body fraction may also reflect other physiological processes that affect the uptake, distribution, or elimination of chemical.

Significance. Pioneering research, using combustion ion chromatography for nontarget analysis of PFASs, has recently shown that large fractions of the extractable organic fluorine in biota $^{53,54}$ and humans ${ }^{55}$ cannot be explained by known PFASs. Because these unidentified PFASs represent a major uncertainty for environmental and human health risk assessment, the identification of priority PFASs, which can be regulated based on their hazard properties, is of great importance. In the current study, we present compelling evidence that F-53B is strongly bioaccumulative in low trophic level fish and provide data in support of its widespread distribution in China. ${ }^{14,15}$ On the basis of its apparent omnipresence and strong bioaccumulation propensity, we hypothesize that F-53B could explain a significant part of previously unidentified organofluorine in biological samples from China. ${ }^{55}$ Although the current production of F-53B has been estimated to 20-30 tons/year and its usage has been restricted to China, ${ }^{14}$ the publicly available information about this compound is attached with large uncertainties. It has also been suggested that the increasing regulatory pressure to substitute PFOS in China may lead to an increasing demand for F-53B in sectors other than metal plating. ${ }^{14}$ Thus, proper use and emission inventories for F-53B are urgently needed to support regulatory actions for this emerging contaminant.

\section{ASSOCIATED CONTENT}

\section{Supporting Information}

The Supporting Information is available free of charge on the ACS Publications website at DOI: 10.1021/acs.est.5b04299.

Additional information regarding quality assurance, quality control, and other materials in Tables S1-S12 and Figures S1-S8 (PDF)

\section{AUTHOR INFORMATION}

\section{Corresponding Author}

*Tel: +86 10 62849239. Fax: +86 10 62849182. E-mail: caiyaqi@rcees.ac.cn.

\section{Notes}

The authors declare no competing financial interest.

\section{ACKNOWLEDGMENTS}

This work was jointly supported by National Key Basic Research Program of China (2015CB931903), the National Natural Science Foundation of China (21537004, 21377145, 21321004), the Strategic Priority Research Program of the Chinese Academy of Sciences (XDB14010201), and the Swedish Research council FORMAS (2014-514). We also acknowledge the constructive comments by two anonymous reviewers that significantly helped to improve the manuscript.

\section{REFERENCES}

(1) Paul, A. G.; Jones, K. C.; Sweetman, A. J. A. First global production, emission, and environmental inventory for perfluorooctane sulfonate. Environ. Sci. Technol. 2009, 43 (2), 386-392.

(2) Wang, Z.; Cousins, I. T.; Scheringer, M.; Buck, R. C.; Hungerbühler, K. Global emission inventories for C4-C14 perfluoroalkyl carboxylic acid (PFCA) homologues from 1951 to 2030, Part I: production and emissions from quantifiable sources. Environ. Int. 2014, $70,62-75$.

(3) Buck, R. C.; Franklin, J.; Berger, U.; Conder, J. M.; Cousins, I. T.; de Voogt, P.; Jensen, A. A.; Kannan, K.; Mabury, S. A.; van Leeuwen, S. P. J. Perfluoroalkyl and polyfluoroalkyl substances in the environment: terminology, classification, and origins. Integr. Environ. Assess. Manage. 2011, 7 (4), 513-541.

(4) Bowman, J. S. Fluorotechnology is critical to modern life: The FluoroCouncil counterpoint to the Madrid Statement. Environ. Health Perspect. 2015, 123 (5), A112-A113.

(5) Giesy, J. P.; Kannan, K. Global distribution of perfluorooctane sulfonate in wildlife. Environ. Sci. Technol. 2001, 35 (7), 1339-1342.

(6) Hansen, K. J.; Clemen, L. A.; Ellefson, M. E.; Johnson, H. O. Compound-specific, quantitative characterization of organic fluorochemicals in biological matrices. Environ. Sci. Technol. 2001, 35 (4), $766-770$. 
(7) Lindstrom, A. B.; Strynar, M. J.; Libelo, E. L. Polyfluorinated compounds: Past, present, and future. Environ. Sci. Technol. 2011, 45 (19), 7954-7961.

(8) 3M Company. 3M Phasing Out Some of its Specialty Materials; 2000. http://www.fluoridealert.org/wp-content/pesticides/3m.may16. 2000.press.release.pdf.

(9) United Nations Environment Programme. Governments unite to step-up reduction on global DDT reliance and add nine new chemicals under international treaty; UNEP, 2009. http://chm.pops.int/ Convention/PressreleaseCOP4Geneva8May2009/tabid/542/ language/en-US/Default.aspx.

(10) U.S. Environmental Protection Agency. 2010/15 PFOA Stewardship Program; 2006. http://www.epa.gov/opptintr/pfoa/ pubs/pfoastewardship.htm.

(11) Calafat, A. M.; Wong, L. Y.; Kuklenyik, Z.; Reidy, J. A.; Needham, L. L. Polyfluoroalkyl chemicals in the US population: Data from the national health and nutrition examination survey (NHANES) 2003-2004 and comparisons with NHANES 1999-2000. Environ. Health Perspect. 2007, 115 (11), 1596-1602.

(12) Glynn, A.; Berger, U.; Bignert, A.; Ullah, S.; Aune, M.; Lignell, S.; Darnerud, P. O. Perfluorinated alkyl acids in blood serum from primiparous women in Sweden: Serial sampling during pregnancy and nursing, and temporal trends 1996-2010. Environ. Sci. Technol. 2012, 46 (16), 9071-9079.

(13) Gomis, M. I.; Wang, Z.; Scheringer, M.; Cousins, I. T. A modeling assessment of the physicochemical properties and environmental fate of emerging and novel per- and polyfluoroalkyl substances. Sci. Total Environ. 2015, 505, 981-991.

(14) Wang, S.; Huang, J.; Yang, Y.; Hui, Y.; Ge, Y.; Larssen, T.; Yu, G.; Deng, S.; Wang, B.; Harman, C. First report of a Chinese PFOS alternative overlooked for 30 years: Its toxicity, persistence, and presence in the environment. Environ. Sci. Technol. 2013, 47 (18), 10163-10170.

(15) Ruan, T.; Lin, Y.; Wang, T.; Liu, R.; Jiang, G. Identification of novel polyfluorinated ether sulfonates as PFOS alternatives in municipal sewage sludge in China. Environ. Sci. Technol. 2015, 49 (11), 6519-6527.

(16) Conder, J. M.; Hoke, R. A.; Wolf, W. D.; Russell, M. H.; Buck, R. C. Are PFCAs bioaccumulative? A critical review and comparison with regulatory criteria and persistent lipophilic compounds. Environ. Sci. Technol. 2008, 42 (4), 995-1003.

(17) $\mathrm{Ng}$, C. A.; Hungerbuhler, K. Bioconcentration of perfluorinated alkyl acids: How important is specific binding? Environ. Sci. Technol. 2013, 47 (13), 7214-7223.

(18) Armitage, J. M.; Arnot, J. A.; Wania, F. Potential role of phospholipids in determining the internal tissue distribution of perfluoroalkyl acids in biota. Environ. Sci. Technol. 2012, 46 (22), 12285-12286.

(19) Armitage, J. M.; Arnot, J. A.; Wania, F.; Mackay, D. Development and evaluation of a mechanistic bioconcentration model for ionogenic organic chemicals in fish. Environ. Toxicol. Chem. 2013, 32 (1), 115-128.

(20) Schmitt, W. General approach for the calculation of tissue to plasma partition coefficients. Toxicol. In Vitro 2008, 22 (2), 457-467.

(21) Ng, C. A.; Hungerbuhler, K. Bioaccumulation of perfluorinated alkyl acids: Observations and models. Environ. Sci. Technol. 2014, 48 (9), 4637-4648.

(22) Roche, H.; Buet, A.; Jonot, O.; Ramade, F. Organochlorine residues in european eel (Anguilla anguilla), crucian carp (Carassius carassius) and catfish (Ictalurus nebulosus) from Vaccarès lagoon (French National Nature Reserve of Camargue) - effects on some physiological parameters. Aquat. Toxicol. 2000, 48, 443-459.

(23) Zhou, Z.; Shi, Y.; Vestergren, R.; Wang, T.; Liang, Y.; Cai, Y. Highly elevated serum concentrations of perfluoroalkyl substances in fishery employees from Tangxun Lake, China. Environ. Sci. Technol. 2014, 48 (7), 3864-3874.

(24) Zhou, Z.; Liang, Y.; Shi, Y.; Xu, L.; Cai, Y. Occurrence and transport of perfluoroalkyl acids (PFAAs), including short-chain
PFAAs in Tangxun Lake, China. Environ. Sci. Technol. 2013, 47 (16), 9249-9257.

(25) Gingerich, W. H.; Pityer, R. A. Comparison of whole-body and tissue blood volumes in rainbow-trout (Salmo-Gairdneri) with ${ }^{125} \mathrm{I}$ bovine serum-albumin and ${ }^{51} \mathrm{Cr}-$ erythrocyte tracers. Fish Physiol. Biochem. 1989, 6 (1), 39-47.

(26) Karlsson-Norrgren, L.; Runn, P. Cadmium dynamics in fish pulse studies with ${ }^{109} \mathrm{Cd}$ in female zebrafish, Brachydanio rerio. J. Fish Biol. 1985, 27 (5), 571-581.

(27) Inoue, Y.; Hashizume, N.; Yakata, N.; Murakami, H.; Suzuki, Y.; Kikushima, E.; Otsuka, M. Unique physicochemical properties of perfluorinated compounds and their bioconcentration of common carp Cyprinus carpio L. Arch. Environ. Contam. Toxicol. 2012, 62, 672680.

(28) Martin, J. W.; Mabury, S. A.; Solomon, K. R.; Muir, D. C. G. Bioconcentration and tissue distribution of perfluorinated acids in rainbow trout (Oncorhynchus mykiss). Environ. Toxicol. Chem. 2003, 22 (1), 196-204.

(29) Berger, U.; Kaiser, M. A.; Karrman, A.; Barber, J. L.; van Leeuwen, S. P. J. Recent developments in trace analysis of poly- and perfluoroalkyl substances. Anal. Bioanal. Chem. 2011, 400 (6), 16251635.

(30) Benskin, J. P.; Ikonomou, M. G.; Woudneh, M. B.; Cosgrove, J. R. Rapid characterization of perfluoralkyl carboxylate, sulfonate, and sulfonamide isomers by high-performance liquid chromatographytandem mass spectrometry. J. Chromatogr. A 2012, 1247, 165-170.

(31) Wang, Z.; Cousins, I. T.; Scheringer, M.; Hungerbühler, K. Fluorinated alternatives to long-chain perfluoroalkyl carboxylic acids (PFCAs), perfluoroalkane sulfonic acids (PFSAs) and their potential precursors. Environ. Int. 2013, 60, 242-248.

(32) Shi, Y.; Vestergren, R.; Xu, L.; Song, X.; Niu, X.; Zhang, C.; Cai, Y. Characterizing direct emissions of perfluoroalkyl substances from ongoing fluoropolymer production sources: A spatial trend study of Xiaoqing River, China. Environ. Pollut. 2015, 206, 104-112.

(33) Han, X.; Snow, T. A.; Kemper, R. A.; Jepson, G. W. Binding of perfluorooctanoic acid to rat and human plasma proteins. Chem. Res. Toxicol. 2003, 16 (6), 775-781.

(34) Bischel, H. N.; Macmanus-Spencer, L. A.; Luthy, R. G. Noncovalent interactions of long-chain perfluoroalkyl acids with serum albumin. Environ. Sci. Technol. 2010, 44 (13), 5263-5269.

(35) Hebert, P. C.; MacManus-Spencer, L. A. Development of a fluorescence model for the binding of medium- to long-chain perfluoroalkyl acids to human serum albumin through a mechanistic evaluation of spectroscopic evidence. Anal. Chem. 2010, 82 (15), 6463-6471.

(36) Zhang, X.; Chen, L.; Fei, X. C.; Ma, Y. S.; Gao, H. W. Binding of PFOS to serum albumin and DNA: Insight into the molecular toxicity of perfluorochemicals. BMC Mol. Biol. 2009, 10, 16.

(37) Luebker, D. J.; Hansen, K. J.; Bass, N. M.; Butenhoff, J. L.; Seacat, A. M. Interactions of flurochemicals with rat liver fatty acidbinding protein. Toxicology 2002, 176 (3), 175-185.

(38) Zhang, L.; Ren, X. M.; Guo, L. H. Structure-based investigation on the interaction of perfluorinated compounds with human liver fatty acid binding protein. Environ. Sci. Technol. 2013, 47 (19), 1129311301.

(39) Lehmler, H.-J.; Bummer, P. M. Mixing of perfluorinated carboxylic acids with dipalmitoylphosphatidylcholine. Biochim. Biophys. Acta, Biomembr. 2004, 1664 (2), 141-149.

(40) Xie, W.; Ludewig, G.; Wang, K.; Lehmler, H. Model and cell membrane partitioning of perfluorooctanesulfonate is independent of the lipid chain length. Colloids Surf., B 2010, 76 (1), 128-136.

(41) Xie, W.; Bothun, G. D.; Lehmler, H.-J. Partitioning of perfluorooctanoate into phosphatidylcholine bilayers is chain lengthindependent. Chem. Phys. Lipids 2010, 163 (3), 300-308.

(42) Pazos, A. J.; Román, G.; Acosta, C. P.; Sánchez, J. L.; Abad, M. Lipid classes and fatty acid composition in the female gonad of pecten maximus in relation to reproductive cycle and environmental variables. Comp. Biochem. Physiol., Part B: Biochem. Mol. Biol. 1997, 117, 393402 . 
(43) Patton, S.; Thomas, A. J. Composition of lipid foams from swim bladders of two deep ocean fish species. J. Lipid Res. 1971, 12, 331335.

(44) Johnson, J. D.; Gibson, S. J.; Ober, R. E. Cholestyramineenhanced fecal elimination of carbon-14 in rats after administration of ammonium [14C]perfluorooctanoate or potassium [14C]perfluorooctanesulfonate. Toxicol. Sci. 1984, 4 (6), 972-976.

(45) Dobrinska, M. R. Enterohepatic circulation of drugs. J. Clin. Pharmacol. 1989, 29 (7), 577-580.

(46) Ankley, G. T.; Kuehl, D. W.; Kahl, M. D.; Jensen, K. M.; Linnum, A.; Leino, R. L.; Villeneuve, D. A. Reproductive and developmental toxicity and bioconcentration of perfluorooctanesulfonate in a partial life-cycle test with the fathead minnow (Pimephales promelas). Environ. Toxicol. Chem. 2005, 24, 2316-2324.

(47) Becker, A. M.; Gerstmann, S.; Frank, H. Perfluorooctanoic acid and perfluorooctane sulfonate in two fish species collected from the Roter Main River, Bayreuth, Germany. Bull. Environ. Contam. Toxicol. 2010, 84 (1), 132-135.

(48) Zhang, L. Z.; Zhang, T.; Zhuang, P.; Zhao, F.; Wang, B.; Feng, G. P.; Song, C.; Wang, Y.; Xu, S. J. Discriminant analysis of blood biochemical parameters at different developmental gonad stages and gender identification for controlled breeding of Amur sturgeon (Acipenser schrenckii, Brandt, 1869). J. Appl. Ichthyol. 2014, 30 (6), 1207-1211.

(49) Kudo, N.; Sakai, A.; Mitsumoto, A.; Hibino, Y.; Tsuda, T.; Kawashima, Y. Tissue distribution and hepatic subcellular distribution of perfluorooctanoic acid at low dose are different from those at high dose in rats. Biol. Pharm. Bull. 2007, 30 (8), 1535-1540.

(50) Liu, C.; Gin, K. Y.; Chang, V. W. C.; Goh, B. P. L.; Reinhard, M. Novel perspectives on the bioaccumulation of PFCs-the concentration dependency. Environ. Sci. Technol. 2011, 45 (22), 9758-9764.

(51) Arnot, J. A.; Gobas, F. A. P. C. A review of bioconcentration factor (BCF) and bioaccumulation factor (BAF) assessments for organic chemicals in aquatic organisms. Environ. Rev. 2006, 14 (4), 257-297.

(52) Beesoon, S.; Martin, J. W. Isomer-Specific binding affinity of perfluorooctanesulfonate (PFOS) and perfluorooctanoate (PFOA) to serum proteins. Environ. Sci. Technol. 2015, 49 (9), 5722-5731.

(53) Loi, E. I. H.; Yeung, L. W. Y.; Taniyasu, S.; Lam, P. K. S.; Kannan, K.; Yamashita, N. Trophic magnification of poly- and perfluorinated compounds in a subtropical food web. Environ. Sci. Technol. 2011, 45 (13), 5506-5513.

(54) Taniyasu, S.; Senthilkumar, K.; Yamazaki, E.; Yeung, L. W. Y.; Guruge, K. S.; Kannan, K.; Yamashita, N. Perfluoroalkyl substances in the blood of wild rats and mice from 47 prefectures in Japan: use of samples from nationwide specimen bank. Arch. Environ. Contam. Toxicol. 2013, 65 (1), 149-170.

(55) Yeung, L. W. Y.; Miyake, Y.; Taniyasu, S.; Wang, Y.; Yu, H.; So, M. K.; Jiang, G.; Wu, Y.; Li, J.; Giesy, J. P.; Yamashita, N.; Lam, P. S. Perfluorinated Compounds and Total and Extractable Organic Fluorine in Human Blood Samples from China. Environ. Sci. Technol. 2008, 42 (21), 8140-8145. 